\title{
Análise preliminar fitoquímica do extrato bruto das folhas de Nephrolepis pectinata
}

\section{Natally Marreiros Gomes ${ }^{1}$, Rosany Lopes Martins ${ }^{2}$ e Sheylla Susan Moreira da Silva de Almeida $^{3}$}

1 Discente do curso de Ciências Farmacêuticas da Universidade Federal do Amapá, Brasil. Email: lanytacarpediem@hotmail.com

2 Doutoranda em Biodiversidade e Biotecnologia - Rede Bionorte pela Universidade Federal do Amapá. Graduada em Ciências Biológicas pela Universidade Federal do Pará. Brasil Email: rosyufpa@gmail.com

3 Doutora em Química pela Universidade Federal de São Carlos. Graduada em Ciências Farmacêuticas pela Universidade Federal do Pará. Professora da Universidade Federal do Amapá, Brasil. Email: sheyllasusan@yahoo.com.br

RESUMO: A Nephrolepis pectinata conhecida popularmente como samambaia paulista, escadinha-do-céu, rabo-de-gato, pertence à família das Davalliaceae. Pela beleza dos arranjos de suas folhas as samambaias são bastante comercializadas no Brasil, no entanto, ainda não foram descritos na literatura estudos sobre seu potencial farmacológico. Desta forma, o objetivo desta pesquisa foi analisar as propriedades fitoquímicas do extrato bruto das folhas de Nephrolepis pectinata. Para realização das análises fitoquímicas foram realizados inicialmente a coleta do vegetal, preparação da exsicata, lavagem, secagem e trituração. Em seguida, a extração pelo método de percolação e por final as análises fitoquímicas. Os resultados preliminares fitoquímicos do extrato bruto das folhas de Nephrolepis pectinata apresentaram resultado positivo para açúcares redutores, fenóis/taninos (taninos catéquinos) e catequinas.

Palavras-chave: Métodos de extração, plantas medicinais, propriedades fitoquímicas.

\section{Analysis preliminary phytochemical raw extract of leaves Nephrolepis pectinata}

ABSTRACT: The Nephrolepis pectinata popularly known as paulista fern, ladder-heaven, cat tail, belongs to the family Davalliaceae. For the beauty of the arrangements of their leaves ferns are quite commercialized in Brazil, however, have not been described in the literature studies on their pharmacological potential. Thus, the objective of this research was to analyze the phytochemical properties of the crude extract of the leaves of Nephrolepis pectinata. To perform the phytochemical analysis were initially made the collection of the vegetable, preparation of voucher specimen, washing, drying and grinding. Then, extraction by percolation method and end the phytochemical analysis. Preliminary results phytochemicals the crude extract of the leaves of Nephrolepis pectinata tested positive for reducing sugars, phenols/tannins (catechins tannins) and catechins.

Keywords: Extraction methods, herbal, phytochemical properties.

\section{INTRODUÇÃO}

O uso das espécies vegetais, com a finalidade de curar doenças, remonta ao início da civilização, a partir do momento em que o homem despertou sua consciência e começou um longo percurso de manuseio, adaptação e modificação dos recursos natu- rais para seu próprio benefício (DI STASI, 1995).

A Organização Mundial da Saúde (OMS) estima que $80 \%$ da população mundial utiliza plantas medicinais como principal recurso no atendimento básico de saúde, seja sob a forma in natura (por opção ou por ser a única alternativa disponível) ou em siste- 
mas de medicina que empregam plantas processadas em formulações medicamentosas, como a medicina chinesa (YUNES; PEDROSA; CECHINEL FILHO, 2001).

A utilização de produtos naturais pela população, tem inclusive recebido incentivos do governo brasileiro, como uma forma de valorizar o conhecimento popular. Como por exemplo, a criação da Política Nacional de Plantas Medicinais e Fitoterápicos, que tem como objetivo garantir à população brasileira o acesso seguro, o uso racional de plantas medicinais e fitoterápicos, promovendo assim o uso sustentável da biodiversidade associada ao desenvolvimento de cadeia produtiva e da indústria nacional (BRASIL, 2006).

Considerando-se a diversidade de espécies vegetais no planeta e a vasta gama de informações etnofarmacológicas sobre o uso de plantas com finalidades terapêuticas, há a necessidade de se desenvolver métodos que facilitem a tarefa de avaliar cientificamente o valor terapêutico das plantas medicinais (BARATTO, 2010).

Apesar dos benefícios observados, muitas reações tóxicas têm sido associadas às plantas. Neste sentido são necessários estudos criteriosos relativos à sua utilização. Diferentes autores (COSTALONGA, 2009; ZUCHETTO, 2014; CAMPOS et al., 2016; MANETTI et al., 2009) têm apontado a importância dos estudos químicos e farmacológicos, em várias espécies vegetais, pela intensa produção de metabólitos secundários, que podem ser medicinais ou tóxicos (BRITO; BRITO, 1993).

A população, de maneira geral, acredita que medicamentos fitoterápicos não possuem efeitos colaterais importantes por serem obtidos de fontes naturais. Contudo, estudos clínicos recentes mostram que essa é uma falsa premissa. Muitas plantas possuem constituintes extremantes tóxicos, como por exemplo, os glicosídeos digitálicos (YUNES; CALIXTO, 2001).

O aumento da demanda pela utilização de plantas medicinais na cura ou prevenção de doenças, o cultivo e/ou o extrativismo dessas plantas torna-se uma alternativa cada vez mais importante na agricultura nacional (REIS; MARIOT; STEENBOCK, 2007).

Os esforços e recursos têm sido utilizados nos últimos anos para a investigação da viabilidade da pesquisa sobre a composição e aplicabilidade de espécies vegetais, tradicionalmente usadas para tratar diferentes sintomas (BARBOSA, 2011).

As pteridófitas são plantas criptógamas vasculares com caule, raiz e folhas (estes últimos ausentes em psilotales), porém sem flores, frutos ou sementes, sendo a reprodução por meio de esporos (RAVEN; EVERT; EICHHORN, 2007).

Além da sua importância biológica, as pteridófitas têm aplicações diversas, uma vez que delas podem ser extraídas substâncias que são utilizadas como medicamentos, nas indústrias de alimentos e cosméticos, como açúcares, proteínas e óleos essenciais (BITTENCOURT; DALLA CORTE; SANQUETTA, 2004). Particularmente, na medicina popular cerca de 220 espécies são utilizadas, sendo que, aproximadamente, 60 espécies são usadas no Brasil (BARROS; ANDRADE, 1997). Na Amazônia, o principal uso é medicinal. Já foram registrados usos para curar diarréia e dor de estômago, dor no corpo, dor de dentes, gripes, dor nos rins, para cicatrizar feridas, desinchar pancadas e também para uso veterinário (ZUQUIM et al., 2008).

O gênero Nephrolepis é tropical e subtropical, com aproximadamente 25-30 es- 
pécies. Sete espécies são nativas das Américas e outras foram introduzidas na região (MICKEL; BEITEL, 1988). No Brasil, o gênero encontra-se composto por nove espécies que se distribuem pelo país inteiro (LOURENÇO, 2012). São plantas terrícolas, epífitas ou rupícolas. Caule ereto a subereto, escamoso (SAKAGAMI, 2006).

A Nephrolepis pectinata pertence à família Davalliaceae (SANTOS et al., 2004) e é conhecida popularmente como samambaia paulista, escadinha-do-céu, rabo-de-gato. Esta espécie se distribui geograficamente no Sul do México, Mesoaméric8.a, Antilhas, Colômbia, Venezuela, Bolívia Peru e Brasil (ROCHA, 2008).

É uma pteridophyta nativa de diversas áreas, principalmente tropical, em que apresenta uma altura de 30 a 40 centímetros. Adapta-se a todas as estações, herbácea, rizomatosa, caule subterrâneo, apresenta folhagem densa, cresce em meio úmidos com sombra e calor, porém apresenta uma resistência a baixas temperaturas. A Nephrolepis pectinata é uma planta ornamental muito indicada para forrações de muros e compõem buquês com flores e residências em geral (EDUARDO; RODRIGUES, 2008; PADRO, 2005).

Os flavonoides das samambaias, de acordo com a literatura, são primariamente restritos a quatro principais grupos: flavonóis, glicosilflavonas, flavonas e flavanonas. Outros flavonoides também são encontrados nas samambaias, porém esporadicamente, como antocianinas, xantonas, flavanonois e as biflavonas (WALLACE, 1989).

As samambaias são utilizadas na medicina popular para o tratamento de picadas de cobra (14 espécies), sendo que as mais utilizadas são: Nephrolepis pectinata, Trichomanes membranaceum, Selaginella praes- tans ou Adiantum obliquum, este último é usado pelos índios Chácobo da Bolívia para tratar diarréia ou reumatismo (BOOM, 1985). Na região amazônica, as samambaias são utilizadas em rituais religiosos por povos tradicionais, sendo empregadas em banhos de felicidade, tanto nas práticas ritualísticas indígenas quanto em cultos afrobrasileiros (TEIXEIRA et al., 2015).

As samambaias são muito utilizadas para ornamentação, no entanto, poucos trabaIhos estão descritos na literatura sobre seu potencial farmacológico. Desta forma, o objetivo deste trabalho foi analisar as propriedades fitoquímicas do extrato seco das folhas de Nephrolepis pectinata e correlacionar com as atividades encontradas na literatura dos metabólitos secundários.

\section{MATERIAIS E MÉTODO}

\section{Material vegetal}

A espécie vegetal Nephrolepis pectinata foi coletada no Município de Macapá, no bairro Zerão. Sendo que alguns ramos foram utilizados para a preparação das exsicatas e o restante foi utilizado para os processos e métodos analíticos em laboratório. A obtenção do extrato, bem como os testes fitoquímicos foram realizados no Laboratório de Farmacognosia e Fitoquímica da Universidade Federal do Amapá (UNIFAP).

\section{Preparação, secagem, moagem, extra-} ção e obtenção do extrato seco

Os ramos de samambaia foram todos lavados com água corrente. Em seguida, foi tirado o excesso de umidade utilizando jornal e, por conseguinte, foi realizada a separação das folhas do caule da planta e colocados para secar. As folhas foram colocadas em estufa de circulação de $\operatorname{ar}(45$ 으) para 
desidratação. Após a secagem das folhas, foi realizado o processo de trituração, até chegar a partículas bem menores, aumentando a superfície de contato e facilitando a extração. Em seguida, foi realizada a extração, usando $70 \mathrm{~g}$ das folhas trituradas com $1.300 \mathrm{~mL}$ de álcool etílico 96\% (EtOH). 0 procedimento foi realizado em duplicata. Para a eliminação do solvente foi utilizado o rotaevaporador, com temperatura em média de $45 \circ$ C e rotação em 60-70 rpm.

\section{Análises fitoquímicos preliminares}

Os testes fitoquímicos realizados foram: Ácidos orgânicos, açúcares redutores, alcaloides, catequinas, fenóis e taninos, flavonoides, saponinas, polissacarídeos e purinas. As análises seguiram as metodologias descritas na Farmacopéia Brasileira (BRASIL, 2010), com algumas adaptações.

\section{Testes para ácidos orgânicos}

$\mathrm{Na}$ pesquisa de ácidos orgânicos no extrato seco das folhas de Nephrolepis pectinata cerca de $3 \mathrm{mg}$ do extrato seco foram dissolvidos em $5 \mathrm{~mL}$ de água destilada e posteriormente filtrado. Foi transferido $2 \mathrm{~mL}$ para um tubo de ensaio e adicionado gotas do reativo de Reativo de Pascová. Sendo a descoloração do reativo indicativo de reação positiva.

\section{Testes para açúcares redutores}

$\mathrm{Na}$ pesquisa de açúcares redutores no extrato seco das folhas de Nephrolepis pectinata foram dissolvidos cerca de $3 \mathrm{mg}$ de extrato seco em $5 \mathrm{~mL}$ de água destilada, a solução foi filtrada e em seguida adicionado ao filtrado $2 \mathrm{~mL}$ de cada reativos FEHLING $A$ e FEHLING B, e posteriormente foi aquecido durante 5 minutos em banho-maria. A reação seria positiva se houvesse a formação de um precipitado vermelho tijolo.

\section{Testes para alcaloides}

Para o teste de alcaloides, diluiu-se cerca de $3 \mathrm{mg}$ de extrato seco em $5 \mathrm{~mL}$ de ácido clorídrico $(\mathrm{HCl})$ concentrado a $5 \%$. A solução foi dividida em três tubos de ensaios, sendo cada um contendo $1 \mathrm{~mL}$ de extrato preparado. Foi filtrado e separado em 3 tubos de ensaio e acrescentado 6 gotas dos reativos de Mayer, Dragendorff e Bouchardat, separadamente, em cada tubo de ensaio.

\section{Testes para catequinas}

Para a análise de catequinas foram utilizados cerca de $3 \mathrm{mg}$ de extrato seco dissolvido em $3 \mathrm{~mL}$ de metanol, um palito de fósforo foi embebedado na solução e evaporado em temperatura ambiente até ficar seco, depois foi umedecido em $\mathrm{HCl}$ concentrado, e em seguida seco em uma chama forte. 0 aparecimento de cor vermelha indica a presença de catequinas.

\section{Testes para fenóis e taninos}

$\mathrm{O}$ extrato seco (3 $\mathrm{mg}$ ) foi dissolvido em $5 \mathrm{~mL}$ de água destilada, em seguida filtrado e adicionado 1 a 2 gotas de solução alcoólica de $\mathrm{FeCl}_{3}$ a 1\%. Sendo que a coloração inicial entre azul e vermelho indica a presença de fenóis, quando comparado com o teste em branco. E precipitados escuro de tonalidade azul (presença de taninos pirogálicos) e verde (presença de taninos catéquicos).

\section{Testes para flavonoides}

O extrato seco (3 $\mathrm{mg}$ ) foi dissolvido em $10 \mathrm{~mL}$ de metanol, em seguida a solução foi filtrada e adicionado 15 gotas de $\mathrm{HCl}$ concentrado e algumas raspas de Magnésio. Sendo o resultado positivo quando ocorre o 
surgimento de coloração rósea na solução.

\section{Teste para saponina}

Para o teste de saponinas foram utilizados $3 \mathrm{mg}$ do extrato seco, e água destilada. O método utilizado foi de saponina espumídica, onde foram dissolvidos $3 \mathrm{mg}$ de extrato seco em $5 \mathrm{~mL}$ de água destilada para solubilização e completado o volume para 10 $\mathrm{mL}$ (qsp). Após este procedimento, a solução foi agitada vigorosamente para formação de espuma e deixada em repouso por um período de $2 \mathrm{~min}$. Sendo que se a camada de espuma permanecer estável por mais de meia hora, o resultado é considerado positivo para saponina espumídica.

\section{Teste para polissacarídeo}

No teste de polissacarídeos cerca de 3 $\mathrm{mg}$ de extrato seco foi dissolvido em $5 \mathrm{~mL}$ de água destilada. Após a dissolução total do extrato na água, o mesmo foi filtrado e adicionado 2 gotas do reagente Lugol. Sendo o aparecimento de coloração azul, indica resultado positivo.

\section{Teste purinas}

Para análise da detecção de purinas, utilizou-se uma cápsula de porcelana, onde foi depositado alguns miligramas do extrato seco e adicionado em seguida 3 gotas de $\mathrm{HCl} 2 \mathrm{~N}$ e duas gotas de $\mathrm{H}_{2} \mathrm{O}_{2}$ a $30 \%$, sendo em seguida evaporado em banho-maria. Devendo formar um resíduo corado de vermelho, onde deveria ser adicionado 3 gotas de $\mathrm{NH} 4 \mathrm{OH} 6 \mathrm{~N}$. Sendo que, o surgimento de coloração violeta, indica a reação positiva.

\section{RESULTADOS E DISCUSSÃO}

Dos testes realizados, os que apresenta- ram resultado positivo foram os de açúcares redutores, fenóis/taninos (taninos catequinos) e catequinas (Tabela 1 ).

Tabela 1. Perfil fitoquímico qualitativo do extrato etanólico da Nephrolepis pectinata

Table 1. Qualitative phytochemical profile of the ethanolic extract from Nephrolepis pectinata

\begin{tabular}{l|c}
\hline \multicolumn{1}{c|}{ Classe dos metabólitos secundários } & Resultados \\
\hline Ácidos orgânicos & - \\
\hline Açúcares redutores & + \\
\hline Alcaloides & - \\
\hline Catequinas & + \\
\hline Fenóis/Taninos & + \\
\hline Flavonoides & - \\
\hline Saponinas & - \\
\hline Polissacarídeos & - \\
\hline Purinas & - \\
\hline
\end{tabular}

Os testes fitoquímicos preliminares das folhas de Nephrolepis pectinata apresentaram resultados positivos para açúcares redutores. Esses metabólitos são fundamentais no processamento de alimentos, especialmente se o produto alimentício contém proteína e for aquecido durante seu preparo, processamento ou conservação. Açúcares redutores são assim denominados por possuírem capacidade de se oxidarem na presença de agentes oxidantes em soluções alcalinas (SILVA et al., 2003). Dentre os açucares redutores estão a glicose e a frutose, o primeiro atua no sistema nervoso central suprindo energia e a sua regulação intestinal protege o organismo em patologias como a diabetes tipo 2, obesidade e síndrome metabólica, já o segundo quando ingerido de forma moderada através de alimentos tem efeitos benéficos a partir de sua utilização como fonte de energia (BARREIROS et al., 2005; ARAÚJO; MARTEL, 2009).

Outro metabólito que apresentou resultado positivo foi a catequina que tem como efeito a redução de gordura corporal baseado no metabolismo dos lipídeos. As catequinas estão presentes também no chá 
verde (LAMARÃO; FIALHO, 2009). A atividade antiulcerogênica deve-se, primordialmente, aos taninos e derivados da catequina. Os derivados da catequina são potentes antioxidantes (mais potentes que a vitamina $C$ ou $E$ para inibir a oxidação in vitro), além de exibirem atividade sobre diferentes radicais livres, tais como o radical superóxido ou peróxido (HO; CHEN; SHI, 1992). A atividade antioxidante dos derivadosda catequina é maior sobre o tubo digestivo, inibindo a lesão de células da mucosa por radicais livres gerados no processo digestivo, evidenciada num modelo in vitro (OLIVEIRA; CUNHA; COLAÇO, 2009).

As análises fitoquímicas também apresentaram resultados positivos para taninos. Os taninos possuem propriedades como adstringentes, cicatrizante e vasoconstritor (LIMA, 2006). Por via interna exercem efeito antidiarréico e anti-séptico; por via externa impermeabilizam as camadas mais expostas da pele e mucosas, protegendo assim as camadas subjacentes (BRUNETON, 1991).

Os mecanismos de ações dos taninos estão relacionados a três fatores: habilidade de complexação com moléculas e também com macromoléculas (proteínas e polissacarídeos); a atividade antioxidante e sequestrante de radicais livres; e a complexação com íons metálicos, como ferro, manganês, cobre, alumínio, cálcio, entre outros (SANTOS; MELLO, 2004). Em processos de cura de feridas, queimaduras e inflamações, os taninos auxiliam formando uma camada protetora (complexo tanino-proteína e/ou polissacarídeo) sobre tecidos epiteliais lesionados, podendo, logo, abaixo dessa camada, o processo curativo ocorrer naturalmente (MONTEIRO, 2005).

\section{CONSIDERAÇÕES}

Assim, os processos de preparo e métodos de extração da planta samambaia, $N$. pectinata juntamente com os testes realizados foram de grande importância na identificação de algumas propriedades fitoquímicas do vegetal. Como os compostos de açúcares redutores, taninos e catequina, que apresentam propriedades referentes a indústrias alimentícias e utilização farmacológica.

\section{REFERÊNCIAS}

ARAÚJO, J. R.; MARTEL, F. Regulação da absorção intestinal de glicose: uma breve revisão. Arquivos de Medicina, Portugal, v. 23, n. 2, p. 35-43, 2009.

BARATTO, L. C. APOCYNACEAE: Himatanthus lancifolius (MULL. ARG.) WOODSON E Rauvolfia sellowii MULL. ARG. 2010. 140 f. Dissertação (Mestrado em Ciências Farmacêuticas)- Universidade Federal do Paraná, Curitiba, 2010.

BARBOSA, W. L. R. et al. (Org.) Etnofarmácia: fitoterapia popular e ciência farmacêutica. 1. Ed. Curitiba: Editora CRV, 2011. 132 p.

BARREIROS, C. R.; BOSSOLAN, G.; TRINDA$D E, P$. E. C. Frutose em humanos: efeitos metabólicos, utilização clínica e erros inatos associados. Revista Nutrição, Campinas, v. 18, n. 3, p. 377-389, maio/jun. 2005.

BARROS, I. C. L.; ANDRADE, L. H. C. Pteridófitas medicinais (samambaias, avencas e plantas afins). Recife: EditoraUniversitária da UFPE. 1997. 223p.

BITTENCOURT, S.; CORTE, D. A. P.; SANQUETTA, C. R. Estrutura da comunidade de pteridophyta em uma floresta ombrófila mista, Sul do Paraná, Brasil. Silva Lusitana, 
Paraná, n. 2, p. 243-254, 2004.

BOOM, B. M. 1985. Ethnopteridology of the Chácobo Indians in Amazonian Bolivia. American Fern Journal, New York, v. 75, n. 1, p. 19-21, jan./mar., 1985.

BRASIL, Ministério da Saúde. Decreto no 5.813 , de 22 de junho de 2006. Política Nacional de Plantas Medicinais e Fitoterápicos. Disponível em: <http://portal.saude.gov.br/ portal/arquivos/pdf/porta riafito.pdf Brasília: Diário Oficial da União 2006.

BRASIL. Farmacopéia Brasileira. 5. ed. Brasília: ANVISA. 2010.

BRITO, A. R. M. S.; BRITO, A. A. S. Forty years of Brazilian medicinal plant research, Journal of Ethnopharmacology, São Paulo, v. 39, n. 1993, p. 53-67, 1992.

BRUNETON, J.; FRESNO, Á. V. Elementos de Fitoquímica y de Farmacognosia. Espanha: Acribia, 1991.

CAMPOS, S. C.; et al. Toxicidade de espécies vegetais. Revista Brasileira de Plantas Medicinais, Campinas, v. 18 , n. 1 , p. 373-382, 2016.

COSTALONGA, S. A. Avaliação dos efeitos alelopáticos e mutagênicos de formas extrativas de Passiflora edulis Sims por meio do bioensaio Allium cepa. 2009. 71 f. Dissertação (Mestrado em Biologia Vegetal) Programa de Pós-Graduação em Biologia Vegetal, Universidade Federal do Espírito Santo, Vitória, 2009.

EDUARDO, C.; RODRIGUES, J. Padrão de distribuição geográfica das espécies de Pteridophyta ocorrentes da Estação de Ecologia Estadual do paraíso Rio de Janeiro, Brasil. Saúde \& Ambiente em Revista, Duque de Caxias, v. 3, n. 1, p 86-95, 2008.

HO, C. T.; et al. Antioxidative effects of polyphenol extract prepared from various chinese Herbs. Preventive Medicine, New Jersey, v. 21, p. 520-525, 1992.
LAMARÃO, C. R.; FIALHO, E. Aspectos funcionais das catequinas do chá verde no metabolismo celular e sua relação com a redução da gordura corporal. Revista de nutrição, Campinas, v. 22, n. 2, p. 257-269, mar./abr., 2009.

LIMA, R. C. Atividade cicatrizante e avaliação toxicológica pré-clínica do fitoterápico sanativo. 2006. 65 f. Dissertação (Mestrado em Ciências Farmacêuticas)- Pós-Graduação em Ciências Farmacêuticas, Universidade Federal de Pernambuco, Recife, 2006. LOURENÇO, S. D. J. Samambaias da estação ecológica do Pau-Brasil, Paraíba, Brasil. 2012. $57 \mathrm{f}$. Trabalho de conclusão de curso (Graduação em Ciências Biológicas e Sociais Aplicadas) - Curso de Ciências Biológicas, Universidade Estadual da Paraíba, João Pessoa, 2012.

MANETTI, M. L.; et al. Metabólitos Secundários da Família Bromeliaceae. Química Nova, Umuarama - PR, v. 32 , n. 7, p. 18851897, 2009.

MICKEL, J. T.; BEITEL, J. M. Pteridophyte Flora de Oaxaca, Mexico. Memoirs of the New York Botanical Garden, 1988.

MONTEIRO, M. J.; et al. Taninos: Uma abordagem da química à Ecologia. Química Nova, São Paulo, v. 28, n. 4, p. 892-896, Sept./Oct. 2005.

OLIVEIRA,S. R.; CUNHA, C. S.; COLAÇO, W. Revisão da Maytenus ilicifolia Mart. ex Reissek, Celastraceae. Contribuição ao estudo das propriedades farmacológicas. Revista Brasileira de Farmacognosia, João Pessoa, v. 19, n. 2, p. 650-659, Apr./June 2009.

PADRO, J. Flora da Reserva Ducke, Amazonas, Brasil: Pterydophyta-Davallia-ceae. Rodriguésia, Rio de Janeiro, v. 56 , n. 86 , p. 3842, 2005.

RAVEN, P.H.; EVERT, R.F.; EICHHORN, S. E. Biologia Vegetal, 7. ed. Rio de Janeiro: 
Guanabara Koogan, 2007.

REIS, S. M.; MARIOT, A.; STEENBOCK, W. Diversidade e domesticação de plantas medicinais. In: SIMÕES, C. M. O.; et al. Farmacognosia: da planta ao medicamento. Porto Alegre: Editora da UFRGS; Florianópolis: Editora da UFSC, 2007. p. 44-73.

ROCHA, L. A. M. Inventário de espécies de Pteridófitas de uma mata de galeria em Alto Paraíso, Goiás, Brasil e morfogênese dos gametófitos de Pecluma ptilodon ( KUNZE) Price e Campyloneurum phyllitidis (L.) C. Presl (Polypodiaceae). 2008. $104 \mathrm{f}$. Dissertação (Mestrado em Botânica) Programa de Pós-Graduação em Botânica, Universidade de Brasília, Brasília- DF, 2008.

SAKAGAMI, R. C. Teridófitas do Parque Ecológico da Klabin, Telêmaco Borba, Paraná, Brasil. 2006. 186 f. Dissertação (Mestrado em Botânica) - Curso de Botânica. Departamento de Ciências Biológicas, Universidade Federal do Paraná, Paraná, 2006.

SANTOS, S. C.; MELLO, J. C. P. Taninos. In: SIMÕES, C. M. O.; et al. Farmacognosia: da planta ao medicamento. Porto Alegre: Editora da UFRGS; Florianópolis: Editora da UFSC, 2007. p. 29-43.

SANTOS, G. M.; et al. Análise florística das pteridófitas do Parque Nacional da Restinga de Jurubatiba, Rio de Janeiro, Brasil. Acta Botanica Brasilica, Rio de Janeiro, v. 18, n. 2, p. 271-280, 2004.

SILVA, N. R.; et al. Comparação de métodos para a determinação de açúcares redutores e totais em mel. Ciência e Tecnologia de Alimentos, Campinas, v. 23, n. 3, p. 337341, set./dez. 2003

TEIXEIRA, G.; PIETROBOM, R. M.; MACIEL, S. Potencial utilitário de licófitas e samambaias: aplicabilidade ao contexto amazônico. Biota Amazônia, Macapá, v. 5, n. 1, p. 6873, abr. 2015.
WALLACE, J. W. Chemosystematic implications of flavonoids and C-Glycosylxanthones in "ferns". Biochemical Systematics and Ecology, Cullowhee, v. 17, n. 2, p. 145-153, mai. 1989.

YUNES, R. A.; PEDROSA, R. C.; CECHI NEL FILHO, V. Fármacos e fitoterápicos: anecessidade do desenvolvimento da indústria de fitoterápicos e fitofármacos no Brasil. Química Nova, Florianópolis- SC, v. 24, n. 1, p. 147-152, jun. 2001.

YUNES, R. A.; CALIXTO, J. B. Plantas medicinais sob a ótica da química medicinal moderna. Chapecó: Artigos, 2001.

ZUQUIM, G.; et al. Guia de Samambaias e Licófitas da REBIO Uatumã- Amazônia Central. Manaus: Áttema Design, 2008.

ZUCHETTO, M. Contribuição ao estudo fitoquímico e atividades biológicas (alelopática, antioxidante e toxicológica in vitro) de Cyathea atrovirens (Langsd. Et Fisch) Domin, Cyatheaceae. 2014. 84 f. Dissertação (Mestrado em Ciências Farmacêuticas) Programa de Pós-Graduação em Ciências Farmacêuticas, Universidade Federal do Paraná, Curitiba, 2014.

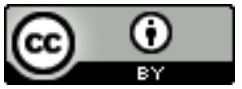
access article distributed under the terms of the Creative Commons Attribution License, which permits unrestricted use, distribution, and reproduction in any medium, provided the original work is properly cited.

Artigo recebido em 18 de março de 2015.

Avaliado em 19 de maio de 2017.

Aceito em 10 de junho de 2017.

Publicado em 18 de agosto de 2017.

\section{Como citar este artigo (ABNT):}

GOMES, Natally Marreiros; MARTINS, Rosany Lopes; ALMEIDA, Sheylla Susan Moreira da Silva de. Análise preliminar fitoquímica do extrato bruto das folhas de Nephrole- 
pis pectinata. Estação Científica (UNIFAP),

Macapá, v. 7, n. 1, p. 77-85, jan./abr. 2017. 